\title{
openheart Role of the central autonomic nervous system intrinsic functional organisation and psychosocial factors in primary microvascular angina and Takotsubo syndrome
}

\author{
Magdalena Maria Cattaneo (10 , ,,2 Emanuele Pravatà, ${ }^{3}$ Micol Provenzi, ${ }^{4}$ \\ Marco Moccetti, ${ }^{5}$ Alain Kaelin, ${ }^{3}$ Isabella Sudano, ${ }^{6}$ Luigi Biasucci, ${ }^{7}$ Camilla Gallino, ${ }^{1}$ \\ Costanzo Limoni, ${ }^{8}$ Carlo Calanchini, ${ }^{9}$ Augusto Gallino, ${ }^{1,10}$ Filippo Crea, ${ }^{7}$ \\ Mattia Cattaneo ${ }^{1,5}$
}

\begin{abstract}
- Additional material is published online only. To view please visit the journal online (http://dx.doi.org/10.1136/ openhrt-2020-001315).
\end{abstract}

To cite: Cattaneo MM, Pravatà $\mathrm{E}$, Provenzi $\mathrm{M}$, et al. Role of the central autonomic nervous system intrinsic functional organisation and psychosocial factors in primary microvascular angina and Takotsubo syndrome. Open Heart 2020;7:e001315. doi:10.1136/ openhrt-2020-001315

MMC and EP contributed equally.

$A G$ and $M C$ contributed equally.

Received 14 April 2020

Revised 12 June 2020

Accepted 15 June 2020

Check for updates

(c) Author(s) (or their employer(s)) 2020. Re-use permitted under CC BY-NC. No commercial re-use. See rights and permissions. Published by BMJ.

For numbered affiliations see end of article.

Correspondence to Dr Magdalena Maria Cattaneo; cattaneo.magdalena@gmail. com

\section{ABSTRACT}

Introduction and objective Dysfunctional central autonomic nervous system network (CAN) at rest may result in aberrant autonomic responses to psychosocial stressors. We hypothesised that patients with primary microvascular angina (MVA) or Takotsubo syndrome (TTS) would exhibit a peculiar functional organisation of the CAN, potentially associated with psychological patterns. Methods Patients underwent a psychosocial evaluation: a clinical diagnostic interview, Millon Clinical Multiaxial Inventory III, State-Trait Anxiety Inventory form Y and Short Form 36 Health Survey (SF-36). The strength of intrinsic functional connectivity ( $\mathrm{FC}$ ) between various nodes of the CAN was investigated using cerebral resting state functional MRI (RS-fMRI).

Results We evaluated 50 (46 women) stable patients: 16 patients with MVA, 17 patients with TTS and 17 patients with previous acute myocardial infarction (AMI). Compared with AMI, patients with MVA showed a lower (higher impairment) SF-36 Body-Pain score (p 0.046) and a higher SF-36 Mental-Health score ( $p$ 0.039). Patients with TTS showed the strongest FC between two nodes of the CAN (sympathetic midcingulate cortex and parasympathetic primary motor area) ( $F 6.25, \mathrm{p} 0.005)$ using RS-fMRI.

Conclusions The study implements an innovative collaborative research among cardiologists, neuroscientists and psychiatrists ('Neuro-psycho-heart Team'). MVA showed a discrepancy between the highest level of self-reported body pain and the best mental health score, which might suggest a mechanism of somatisation. TTS exhibited an increased functional integration between two areas of the CAN involved in interoceptive pain awareness and negative emotional status. We implemented an innovative research collaboration among cardiologists, neuroscientists and psychiatrists. These data are hypothesis generating and suggest potential prospective investigations on pathophysiology and implementation of psychotherapy and stress-reducing techniques as therapeutic strategies.

Trial registration number NCT02759341.

\section{Key questions}

What is already known about this subject?

- Primary microvascular angina (MVA) and Takotsubo syndrome (TTS) are cardiovascular conditions, which share features concerning microvascular dysfunction and potential interaction with the central autonomic nervous system network (CAN) in the presence of normal coronary arteries. Convincing evidence exists that psychosocial stress perturbs the CAN, which facilitates autonomic response imbalance and subsequent endothelial dysfunction, which play a major role in the pathophysiology of both MVA and TTS.

What does this study add?

- We report, for the first time, an extensive psychosocial analysis, while investigating distinguishing features of functional organisation of the CAN by functional MRI in MVA and TTS during stable clinical phase, suggesting potential neuropsychiatric predisposing mechanisms without conceivable acute phase confounders following either the acute TTS/ acute myocardial infarction event or acute changes in clinical status in MVA. Our data suggest complex mechanisms of somatisation that may explain the discrepancy between self-reported mental health and the level of body pain in MVA, possibly resulting in chronic refractory symptoms. Moreover, TTS exhibits a stronger functional integration between two specific brain regions involved in pain experience regulation and visceromotor control that could represent a pathophysiological mechanism of vulnerability to TTS.

\section{INTRODUCTION}

Primary microvascular angina (MVA) and Takotsubo syndrome (TTS) are, respectively, chronic and acute cardiac conditions, whose clinical presentations mimic those of typical 


\section{Key questions}

How might this impact on clinical practice?

- This hypothesis-generating study implements an innovative collaboration among cardiologists, neuroscientists and psychiatrists evaluating patients with microvascular angina (MVA) and Takotsubo syndrome (TTS) as a 'Neuro-psycho-heart Team'. Since no evidence-based therapies were found to be effective in either reducing anginal symptoms in MVA, and reducing recurrence in TTS, it is feasible that future therapeutic protocols should investigate, whether specific central autonomic nervous patterns, as well as symptoms and recurrence, could be modified by behavioural and stress-reducing techniques.

obstructive coronary artery disease (CAD), in the presence of normal or almost normal coronary arteries. ${ }^{12}$ The pathophysiology of these two conditions seems to share some features concerning microvascular dysfunction and the potential interaction with central autonomic nervous system network (CAN). A few studies have investigated psychiatric disorders ${ }^{3}$ and quality of life $^{35}$ in patients with MVA, but these studies often enrolled patients with non-cardiac causes of chest pain. Various studies have shown an association between chronic psychiatric disorders and TTS, ${ }^{6-9}$ but a comprehensive clinical diagnostic psychiatric and psychosocial evaluation of these patients was lacking.

Nonetheless, convincing evidence exists that psychosocial stress perturbs the central nervous system in areas collectively called the CAN. ${ }^{10}$ The CAN includes two distinct subdivisions that are linked to the sympathetic (sCAN) and parasympathetic (pCAN) regulatory response to external stressors. ${ }^{11}$ Dysregulation of the CAN network has been assumed to facilitate autonomic response imbalance and subsequent endothelial dysfunction, ${ }^{12}$ which have been found to play a major role in the pathophysiology of both MVA and TTS. ${ }^{13} 14$ Cerebral functional magnetic resonance imaging (fMRI) can evaluate the organisation and the strength of the functional connectivity (FC) to characterise the areas comprising the CAN. ${ }^{111516}$ Here, we hypothesise that a dysfunctional organisation of the CAN at rest may predispose subjects to aberrant autonomic responses to stressful psychosocial and environmental stimuli in MVA and TTS. The assessment of potential differences in the functional organisation of the CAN, combined with evaluation of psychiatric and psychosocial patterns, may show the link between psychosocial distress and the clinical cardiac manifestation in MVA and TTS. This could substantiate the importance of future potential investigations on the CAN pathophysiology beside the effects of psychotherapy and stress-reducing techniques for these conditions. Therefore, the purpose of the present study was to investigate potential differences in the functional organisation of the CAN, using resting state-(RS) fMRI, and the psychological patterns of patients diagnosed with MVA or TTS compared with patients with a previous acute myocardial infarction (AMI).

\section{METHODS \\ Population}

Between February 2016 and November 2017, we prospectively enrolled patients referrer to or followed up either at the cardiovascular department of the Hospital of San Giovanni (Bellinzona, Switzerland) or at the Cardiocentro Ticino (Lugano, Switzerland). Subjects were enrolled into three groups that fulfilled the following inclusion criteria: patients with a diagnosis of $\mathrm{MVA}^{1}$; patients with a history of TTS at least 6 months after the event ${ }^{2}$; and patients with AMI related to acute myocardial ischaemia (either with or without persistent ST-segment elevation AMI) at least 6 months after the event. ${ }^{17}$ The study approval and enrolment periods preceded the publications of recent international consensus documents on diagnostic criteria for MVA and TTS. ${ }^{18} 19$ MVA diagnostic criteria were as follows ${ }^{1}$ : stable angina, predominantly induced by effort; findings compatible with myocardial ischaemia from either ECG and/or imaging functional tests; normal (or near normal) coronary arteries at angiography; no structural or coronary cardiac disease (vasospstic angina, cardiomyopathy, valvular heart disease). TTS diagnostic criteria were as follows ${ }^{2}$ : transient hypokinesis, akinesis or dyskinesis of the left ventricular segments with or without apical involvement that extend beyond a single epicardial vascular distribution; with or without a recognisable stressful trigger; absence of obstructive coronary disease or angiographic evidence of acute plaque rupture; new electrocardiographic abnormalities or modest elevation in cardiac troponin; absence of pheochromocytoma and myocarditis.

The exclusion criteria were as follows: refused informed consent, unable to either participate or provide written informed consent, short-term survival ( $<1$ year), any acute or decompensated medical, neurological, psychiatric conditions in the last 6 months requiring either inpatients or unscheduled outpatients management (with exception of those listed in the inclusion criteria).

\section{Study schedule}

Patients underwent three visits, as described in figure 1 . RS-fMRI processing and analysis were blinded.

\section{Psychiatric and psychosocial evaluation}

The first part included a comprehensive psychiatric assessment based on the Association for Methodology and Documentation in Psychiatry, eighth edition (AMDP8) System..$^{20}$ During the same visit, three validated questionnaires were administered: the Millon Clinical Multiaxial Inventory III (MCMI-III), ${ }^{21}$ the State-Trait Anxiety Inventory, Form Y (STAI-Y) ${ }^{22}$ and the Short Form 36 Health Survey (SF-36), which is related to quality of life. ${ }^{23}$

The MCMI-III provides a measure of numerous personality disorders and psychiatric clinical syndromes based on the Diagnostic and Statistical Manual of Mental 


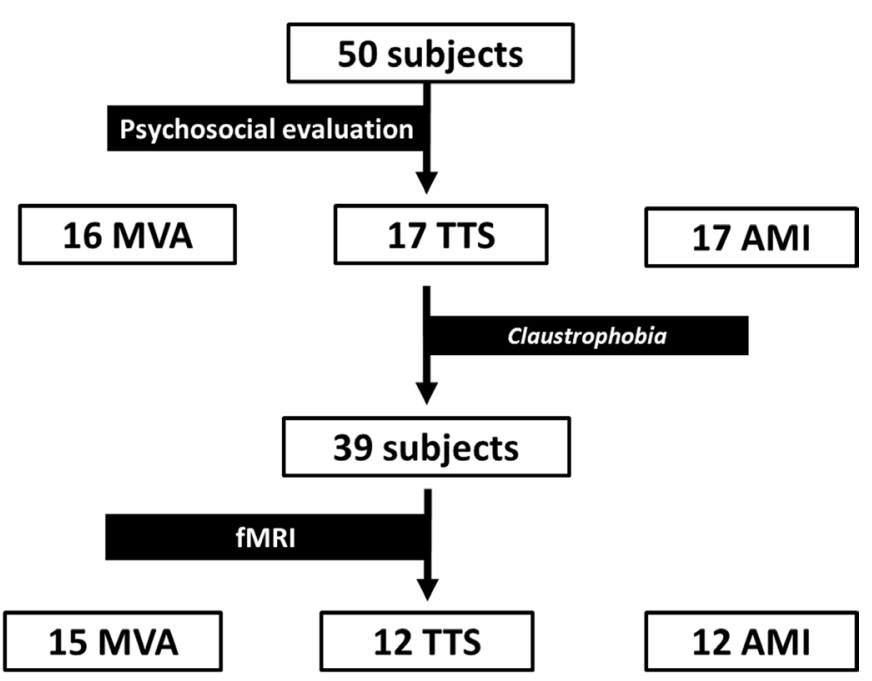

Figure 1 Study schedule and drop-outs. The figure shows the 3-visit study schedule along with the distribution of dropouts. AMI, acute myocardial infarction; fMRI, functional magnetic resonance imaging; MVA, microvascular angina; TTS, Takotsubo syndrome.

Disorders-IV. It consists of true-false questions comprising scales, with higher scores indicating greater clinical significance of the disorder: 14 personality disorder scales, 10 psychiatric clinical syndrome scales and 5 control scales to determine a patient's response style. As the number of scales in the pathological range was negligible, we decided to simplify the categories (online supplementary material).

The STAI-Y is a self-report psychological questionnaire used to evaluate the level and characteristics of anxiety. ${ }^{24}$ The STAI-Y measures state anxiety (SA) and trait anxiety (TA). The STAI-Y consists of 40 items scored on a fourpoint scale, from 1 to 4 , measuring the increasing frequency or intensity of each item..$^{22} 24$

The SF-36 is also a self-reported survey, which assesses physical- and mental-health related quality of life (HRQoL).$^{25}$ Weighted sums of the scores of 11 sets of questions are transformed into percentage scales for 10 different psychosocial issues and two main indices, a Physical-Health index and a Mental-Health index, where higher scores indicate a better state of health (onine supplementary material).

\section{RS FC cerebral fMRI}

RS-fMRI measures spontaneous fluctuations of the bloodoxygen-level-dependent signal in the blood. Changes in oxygen consumption affect the fraction of blood deoxygenated haemoglobin, which is subsequently used as a natural contrast agent. ${ }^{26}$ The fMRI signal reflects the effect of neural activity on dynamic changes in cerebral blood flow and cerebral rate of oxygen metabolism (online supplementary material).

\section{Protocol}

Participants abstained from smoking, alcohol, coffee and tea for 24 hours preceding the exam and rested for
15 min in a quiet, controlled environment before being scanned. The participants were instructed to lie quietly in the MRI scanner with their eyes closed and to let their mind wander. The anxiety level of each participant after entering the MRI scanner was measured by a self-reported scale ranging from 0 (absent) to 9 (maximum). All MRI scans were acquired with a 3.0T Siemens 'Skyra' scanner (Siemens AG, Erlangen, Germany), using a previously published protocol ${ }^{16}$ (online supplementary material).

\section{Data analysis and management}

The processing pipeline, embedded in the CONN V.17 FC toolbox software (https://www.nitrc.org/projects/ conn/), was used for MRI data preparation and analysis. ${ }^{27}$ Details of the image acquisition protocol and preprocessing steps are provided in the online supplementary material. Three-millimetre radius regions of interest (ROIs) were drawn in Montreal Neurological Institute standard space for each of the CAN nodes. Individual nodes were located according to the previous pooled meta-analysis performed by Beissner et $a l^{11}$ (online supplementary material). Three levels of connectivity assessment were performed after estimating the correlation coefficients of each ROI pair and transforming them to $\mathrm{z}$ scores to permit parametric analyses:

1. Within-network mean FC $(\mathrm{mFC})$ of the CAN-the sCAN and pCAN networks were analysed separately, by averaging all the connectivity scores between each network ROI pair. ${ }^{15} 28$

2. Between-networks mFC of the sCAN and pCAN subdivisions were analysed by estimating the average of the connectivity scores between each ROI from one network and each ROI from the other network. ${ }^{1528}$

3. Pair-wise FC analysis was done by calculating the connectivity scores of each ROI pair, both within CAN, sCAN and pCAN, as well as between the sCAN and pCAN. This provided information about the RS-FC strength between specific network nodes.

\section{Additional measures}

Enhanced pain perception may be a crucial mechanism in patients with MVA and TTS; thus, the forearm ischaemic pain test (FIP) was performed to assess general sensitivity to pain 12 months later ${ }^{29}$ (online supplementary material).

\section{Statistics}

All statistical analyses were performed with IBM-SPSS software, V.22 (IBM). Continuous data are presented as mean and SD, while categorical data are presented as numbers and percentages. Comparisons between groups were performed by Student's t-test and analysis of variance (ANOVA) for continuous data, and the $\chi^{2}$ test for categorical data. Statistical significance was set at a rounded two-tailed $\mathrm{p}<0.05$. RS-FC data (expressed as connectivity z-scores) generated by CONN were submitted to SPSS to perform between-group comparisons. Age-adjusted ANOVA was used to test group effects 
on: (1) within-network mFC; (2) between-network mFC and (3) pair-wise FC. Post hoc independent sample t-tests were used when appropriate. Connectivity analyses were adjusted for multiple comparisons using the false discovery rate method. ${ }^{30}$

\section{Patient and Public Involvement}

Patients with MVA and TTS in regular follow-up at our cardiovascular centres often expressed their feeling about existence of a possible relation between brain and heart, that may explain their cardiac chronic symptoms or an acute event, respectively. They underlined a growing need to understand better pathophysiology of their cardiac condition. Patients appreciated and supported an innovative collaboration between cardiologists, neuroscientists and psychiatrists working as a 'Neuro-psycho-heart Team', investigating these disorders, and going further than a simplistic concept of a single-organ disease. In this way, patients were involved in development of the research question. They were not involved at the other stages of the study.

\section{RESULTS}

\section{Study population}

We prospectively enrolled 50 participants, who were divided into three groups: 16 patients with MVA, 17 patients with TTS and 17 patients with AMI (figure 1). As shown in online supplementary tables S1 and S2, participants were matched for age-sex-cardiovascular risk factors. There was no significant difference in psychotropic medications prescribed (table 1), but we found predictable differences in cardiovascular therapies (table 1).

\section{Psychiatric and psychosocial evaluation}

The SF-36 results showed that patients with MVA had a lower Body-Pain score (52.69 vs 69.47; $\mathrm{p}=0.046$ ) and a higher Mental-Health score (76.75 vs 63.76; $\mathrm{p}=0.039$ ) in comparison to the patients with AMI (online supplementary table S3). The SF-36 results also showed that a greater percentage of patients with MVA reported a Mental-Health score higher than the age-sex-matched general population, compared with the patients with TTS and AMI (93.8 vs $76.5 \%$ vs $64.7 \%$; $\mathrm{p}=0.056$ ).

The majority of subjects with TTS $(76 \%)$ reported one acute stressful event that was identified as a trigger, in contrast to patients with AMI, who had no one evident trigger. In TTS group emotional triggers were more frequent than physical ones, $64 \%$ vs $12 \%$, respectively. In $24 \%$ of TTS patients, no evident trigger was identified.

We found no differences in most of the demographic variables from the AMDP-8 System (online supplementary table S2). The results from the MCMI-III, STAI-Y and FIP did not significantly differ among groups (online supplementary table S4-S6). However, we observed a $60 \%$ prevalence rate of obsessive-compulsive personality traits (OCPT) in the entire study sample, which did not differ by group or gender.

\section{RS FC cerebral fMRI}

Patients' self-reported anxiety while in the MRI scanner did not differ among groups (MVA=1.5, TTS=1.6, $\mathrm{AMI}=0.9 ; \mathrm{p} 0.422)$. We found no significant differences with regard to the $\mathrm{mFC}$ within the entire $\mathrm{CAN}(\mathrm{F}=0.19$; $\mathrm{p}=0.80)$, the $\operatorname{sCAN}(\mathrm{F}=0.89 ; \mathrm{p}=0.42)$ and $\mathrm{pCAN}(\mathrm{F}=0.55$; $\mathrm{p}=0.58$ ) subdivisions or between-networks, that is, sCAN$\mathrm{pCAN}(\mathrm{F}=0.18 ; \mathrm{p}=0.83$ ) (figure 2).

The pair-wise analyses showed that patients with TTS exhibited stronger connectivity between the sympathetic midcingulate cortex (sCAN-MCC) and parasympathetic primary motor area (pCAN-PM), compared with patients with AMI ( $\mathrm{p}=0.022)$ and MVA $(\mathrm{p}=0.050)$ (figure 3$)$.

\section{Psychosocial investigation and RS FC cerebral fMRI}

As we found a stronger FC between sCAN-MCC and pCAN-PM in patients with TTS, compared with patients with MVA and AMI, we correlated (using Pearson's r) the corresponding mFC z-scores with the SF-36 and STAI-Y scores. A borderline negative correlation was found with the SF-36 Body-Pain score ( $\mathrm{r}=-0.315, \mathrm{p}=0.051$ uncorrected), which means that the stronger $\mathrm{mFC}$ between the sCAN-MCC and pCAN-PM was related to the perception of worse physical pain.

\section{DISCUSSION}

To the best of our knowledge, this is the first study of stable patients with a history of TTS, AMI or MVA that evaluated potential differences in psychosocial and psychiatric profiles, while investigating the distinguishing features of the intrinsic functional organisation of the CAN using fMRI. By performing an analysis of both psychosocial features and FC of the CAN, we tried to define new potential pathological pathways in cardiovascular diseases that are not explained by existing evidence regarding CAD.

In this analysis, the patients with MVA showed a higher level of self-reported body pain, as assessed by the SF-36, while displaying better self-reported mental health, compared with patients with AMI and the age-matched and sex-matched general population. Moreover, patients with TTS exhibited increased RS FC in specific areas of the CAN involved in interoceptive pain awareness, negative emotional status and visceral sympathetic activity (sCAN-MCC) and parasympathetic regulation (pCANPM), long after the event (44.6 \pm 20.2 months).

Our findings are hypothesis-generating and require further confirmation. However, these data underlined a growing need to understand pathophysiology better. We implemented an innovative research collaboration among cardiologists, neuroscientists and psychiatrists working as a 'Neuro-psycho-heart Team' overcoming a simplistic concept of a single-organ disease. Since no evidence-based cardiovascular therapies have been found to be effective in either reducing anginal symptoms in MVA and reducing recurrence in TTS, it is feasible that future therapeutic protocols should investigate, whether specific CAN patterns, as well as symptoms and 
Table 1 Characteristics of the sample

\begin{tabular}{|c|c|c|c|c|c|c|c|}
\hline & \multicolumn{2}{|c|}{ MVA } & \multicolumn{2}{|l|}{ TTS } & \multicolumn{2}{|c|}{ AMI } & $\mathrm{CHI} 2$ \\
\hline \multicolumn{8}{|l|}{ General } \\
\hline Mean age & 68.8 & & 71.1 & & 65.8 & & $\mathrm{P}=0.15$ \\
\hline Sex (female) & 15 & $93.8 \%$ & 16 & $94.1 \%$ & 14 & $82.4 \%$ & $P=0.43$ \\
\hline Retired & 14 & $87.5 \%$ & 16 & $94.1 \%$ & 13 & $76.5 \%$ & $\mathrm{P}=0.326$ \\
\hline Right handed & 16 & $100.0 \%$ & 17 & $100.0 \%$ & 17 & $100.0 \%$ & NA \\
\hline Time from TTS or AMI (months) & & NA & & $44.6 \pm 20.2$ & & $80.0 \pm 54.9$ & NA \\
\hline Angina CCS class (maximal) & & & & & & & NA \\
\hline I & 0 & $0.0 \%$ & & NA & 1 & $5.6 \%$ & \\
\hline$\|$ & 7 & $41.2 \%$ & & & 0 & $0.0 \%$ & \\
\hline III & 3 & $17.6 \%$ & & & 0 & $0.0 \%$ & \\
\hline IV & 7 & $41.2 \%$ & & & 0 & $0.0 \%$ & \\
\hline Angina during & & & & & & & NA \\
\hline Emotional stress & 3 & $17.6 \%$ & & NA & 0 & $0.0 \%$ & \\
\hline Environmental exposure & 2 & $11.8 \%$ & & & 0 & $0.0 \%$ & \\
\hline Angina frequency & & & & & & & NA \\
\hline Daily & 2 & $11.8 \%$ & & NA & 0 & $0.0 \%$ & \\
\hline$<1 /$ weekly & 10 & $58.8 \%$ & & & 1 & $5.6 \%$ & \\
\hline$>1 /$ weekly & 5 & $29.4 \%$ & & & 0 & $0.0 \%$ & \\
\hline NYHA class & & & & & & & NA \\
\hline I & 13 & $76.5 \%$ & & NA & 2 & $11.1 \%$ & \\
\hline$\|$ & 4 & $23.5 \%$ & & & 15 & $88.9 \%$ & \\
\hline LVEF (\%) & & NA & & NA & & $58.0 \pm 10.1$ & NA \\
\hline \multicolumn{8}{|l|}{ Classic cardiovascular risk factors } \\
\hline Hypertension & 10 & $62.5 \%$ & 8 & $47.10 \%$ & 14 & $82.4 \%$ & $\mathrm{P}=0.10$ \\
\hline Hyperlipidaemia & 9 & $56.3 \%$ & 15 & $88.2 \%$ & 16 & $94.1 \%$ & $P=0.014^{\star}$ \\
\hline Diabetes mellitus & 1 & $6.3 \%$ & 2 & $11.8 \%$ & 4 & $23.5 \%$ & $\mathrm{P}=0.34$ \\
\hline Active smoker & 1 & $6.3 \%$ & 2 & $11.8 \%$ & 2 & $11.8 \%$ & $\mathrm{P}=0.83$ \\
\hline Previous smoker & 5 & $31.3 \%$ & 5 & $29.4 \%$ & 8 & $47.1 \%$ & $\mathrm{P}=0.5$ \\
\hline Family history & 12 & $75.0 \%$ & 9 & $52.9 \%$ & 14 & $82.4 \%$ & $P=0.15$ \\
\hline \multicolumn{8}{|l|}{ Psychiatric medical history } \\
\hline Known major depression & 0 & $0.0 \%$ & 1 & $5.9 \%$ & 2 & $11.8 \%$ & $\mathrm{P}=0.36$ \\
\hline Known anxiety disorder & 0 & $0.0 \%$ & 4 & $23.5 \%$ & 2 & $11.8 \%$ & $\mathrm{P}=0.11$ \\
\hline Known psychotic disorder & 0 & $0.0 \%$ & 0 & $0.0 \%$ & 0 & $0.0 \%$ & NA \\
\hline \multicolumn{8}{|l|}{ Psychotropic medications } \\
\hline Antiepileptic & 0 & $0.0 \%$ & 0 & $0.00 \%$ & 0 & $0.0 \%$ & NA \\
\hline Atypical neuroleptic & 1 & $6.3 \%$ & 1 & $5.90 \%$ & 0 & $0.0 \%$ & $P=0.584$ \\
\hline Benzodiazepine & 6 & $37.5 \%$ & 7 & $41.2 \%$ & 5 & $29.4 \%$ & $P=0.766$ \\
\hline Non-BDZ hypnotic & 1 & $6.3 \%$ & 1 & $5.9 \%$ & 1 & $\mathrm{P}=5.9 \%$ & $P=0.999$ \\
\hline SNRI & 0 & $0.0 \%$ & 1 & $5.9 \%$ & 1 & $5.9 \%$ & $\mathrm{P}=0.613$ \\
\hline SSRI & 0 & $0.0 \%$ & 3 & $17.6 \%$ & 4 & $23.5 \%$ & $\mathrm{P}=0.13$ \\
\hline III generation neuroleptic & 0 & $0.0 \%$ & 0 & $0.0 \%$ & 0 & $0.0 \%$ & NA \\
\hline Tricyclic antidepressant & 1 & $6.3 \%$ & 1 & $5.9 \%$ & 0 & $0.0 \%$ & $\mathrm{P}=0.584$ \\
\hline Typical neuroleptic & 0 & $0.0 \%$ & 0 & $0.0 \%$ & 1 & $5.9 \%$ & $\mathrm{P}=0.371$ \\
\hline 5-HT1A partial agonist & 0 & $0.0 \%$ & 0 & $0.0 \%$ & 0 & $0.0 \%$ & NA \\
\hline IMAO & 0 & $0.0 \%$ & 0 & $0.0 \%$ & 0 & $0.0 \%$ & NA \\
\hline
\end{tabular}

AMI, acute myocardial infarction; BDZ, benzodiazepine; CCS, Canadian cardiovascular society grading of angina; $\mathrm{CHI}$, $\mathrm{CHI}$ square test; 5-HT1A, serotonin 1A receptor; IMAO, monoamine oxidase inhibitors; LVEF, fractional left ventricular ejection fraction; MVA, microvascular angina; NA, not applicable; NYHA, New York Heart Association Functional Classification; SNRI, serotonin-norepinephrine reuptake inhibitors; SSRI, selective serotonin reuptake inhibitors; TTS, Takotsubo syndrome. 


\section{A}

$F=0.19 . p=0.82$

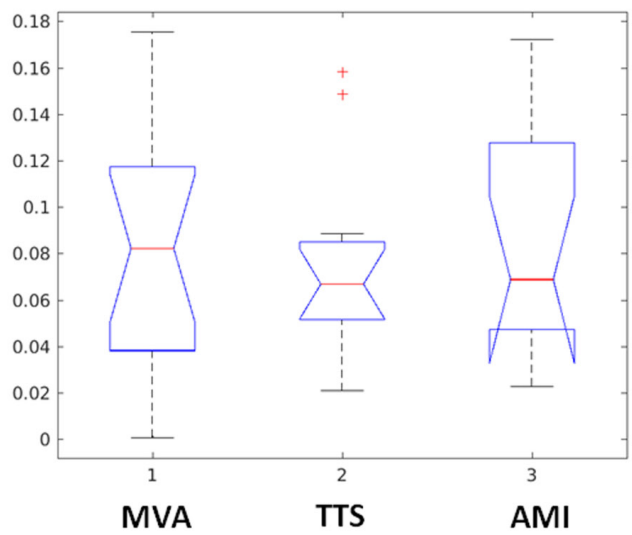

C $F=0.89 . p=0.42$

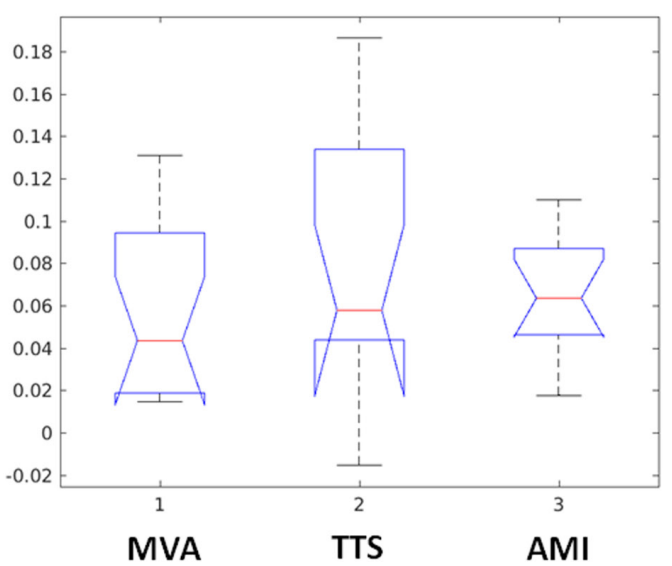

B

$F=0.55 . p=0.58$

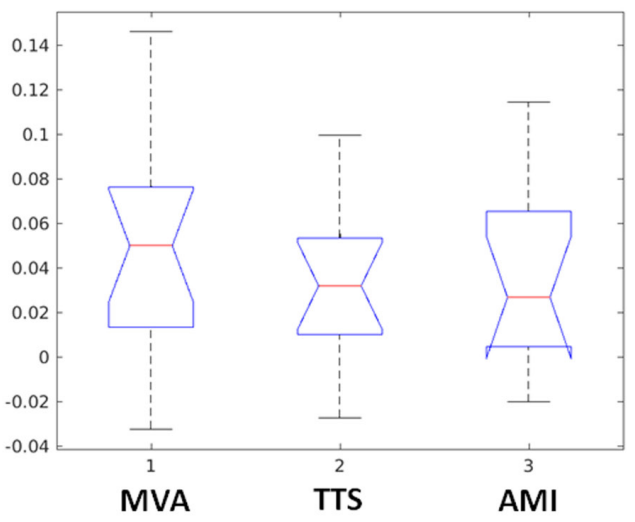

D $F=0.18 . p=0.83$

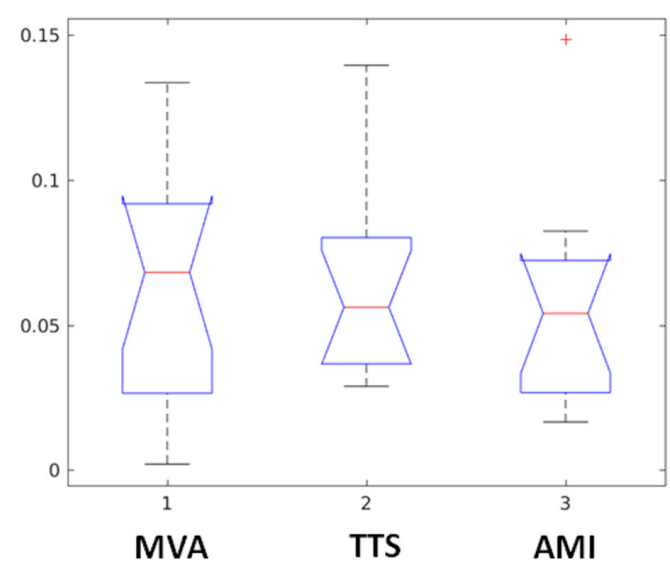

AMI

Figure 2 Main analysis of resting state functional connectivity (FC) using functional MRI (fMRI). The resting state FC measured by fMRI did not differ among the groups. The box-plots show Pearson's correlation coefficients as follows: (A) whole central autonomic nervous system network (CAN) mean FC. (B) Sympathetic network (sCAN) mean FC. (C) Parasympathetic network (pCAN) mean FC. (D) sCAN-pCAN between-networks mean FC. AMI, acute myocardial infarction; MVA, microvascular angina; TTS, Takotsubo syndrome.

recurrence, could be modified by behavioural and stressreducing techniques.

\section{Psychiatric and psychosocial evaluation}

Pain represents both a selective issue and a major concern impairing HRQoL in patients with MVA, which is a chronic syndrome often characterised by resistant chest pain and functional disability. ${ }^{18} 31$ Nonetheless, few studies have investigated the HRQoL in patients with MVA. ${ }^{35}$ Patients with MVA showed poorer scores on various HRQoL scales, particularly on scales inquiring pain and mental health, compared with patients with CAD. In contrast, we found no difference in HRQoL between patients with AMI without chronic heart failure and patients with TTS. ${ }^{32}$ Despite a higher score for body pain, not restricted to angina, patients with MVA exhibited better self-reported mental health, as compared with patients with AMI. Here, we hypothesise somatisation phenomena, which result in better self-reported mental health. Dickinson $e t a l^{33}$ observed that patients affected by various somatisation phenomena report considerable physical impairment, despite a mental health score slightly lower than those of the general population. It is noteworthy that a few studies have reported a higher prevalence of somatoform disorders and a potentially heightened pain perception in patients with MVA, compared with patients with CAD. ${ }^{13}$ Forearm ischaemic pain perception did not differ in patients with MVA; thus, we cannot determine whether these potential misperceptions are specific cardiac abnormalities or symptoms of a general pain disorder.

Unlike our study, the international registry of patients with TTS shows a higher prevalence of previous and chronic psychiatric disorders compared with patients with AMI, while previous investigations of patients with 

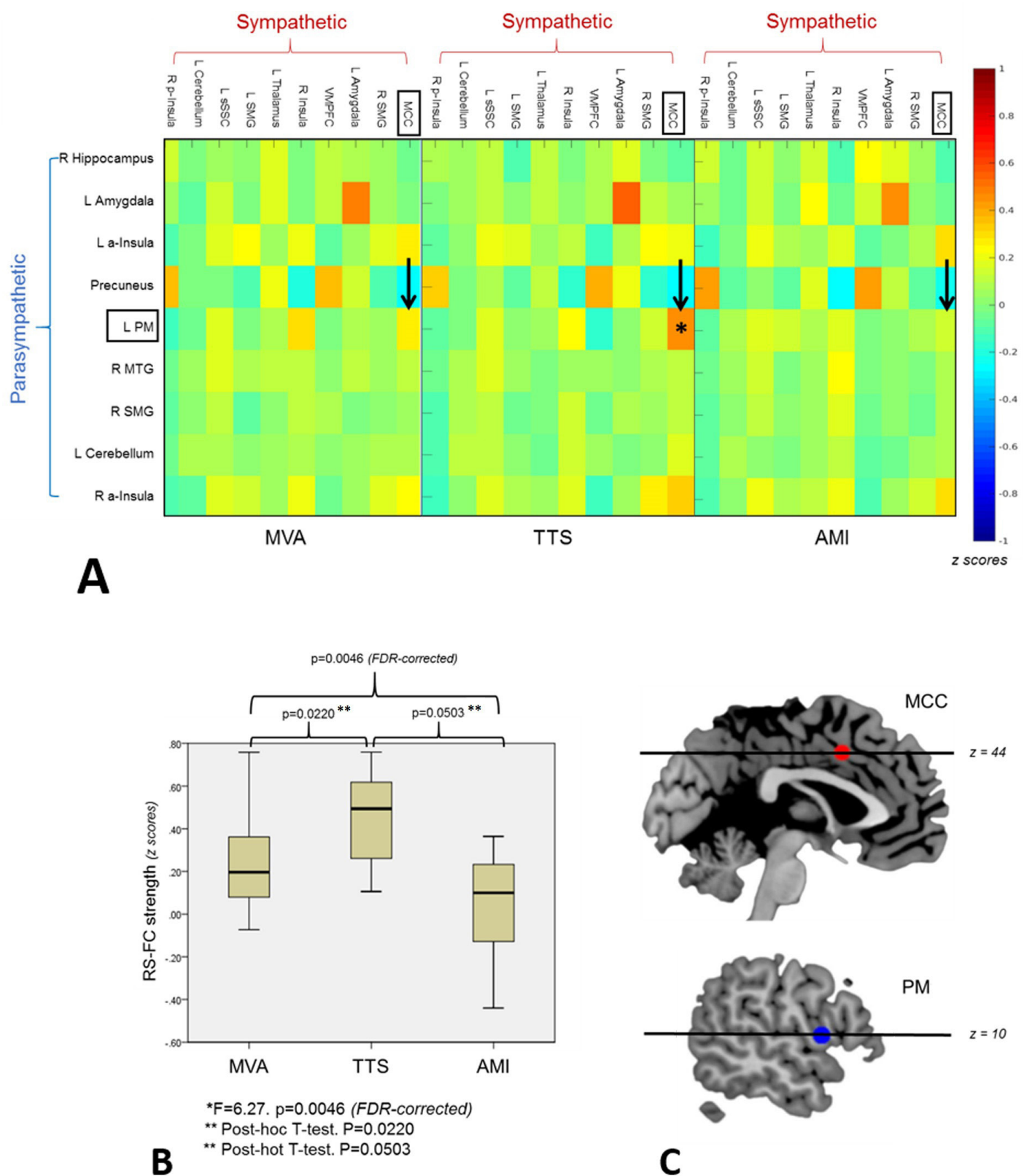

C

Figure 3 Exploratory analysis of resting state (RS) functional connectivity (FC) using functional MRI (fMRI). Plots and matrices represent colour-coded region of interest (ROI)-to-ROI pair FC of sympathetic-parasympathetic synchronisation. (A) The colour indicates the z-score between each pair of ROls. As shown (*), the Takotsubo syndrome (TTS) group showed a stronger connectivity compared with acute myocardial infartion (AMI) group between the SCAN midcingulate cortex (sCAN-MCC) and the $p C A N$ primary motor area ( $P C A N-P M)(p=0.022)$ and a borderline stronger connectivity compared with microvascular angina (MVA) group ( $p=0.050$ ). A full list of the fMRI regions of interest based on Beissner et $a l^{11}$ is in the online supplementary material. (B) The figure shows the location of the two above-mentioned areas in the central nervous system (sCAN-MCC, pCAN-PM). a-Insula, anterior insula; CAN, central autonomic nervous; FDR, false discovery rate; L, left; MCC, midcingulate cortex; MVA, microvascular angina; MTG, medial temporal gyrus; pCAN, parasympathetic CAN; p-Insula, posterior insula; PM, primary motor area; R, right; SCAN, sympathetic CAN; SMG, supramarginal gyrus; SSSC, secondary somatosensory cortex; VMPFC, ventromedial prefrontal cortex.

MVA provided inconsistent results. ${ }^{34} 34$ Therefore, the small sample size, the frequent use of psychotropic medications and the prevalence of OCPT that was higher than expected from clinical practice ${ }^{35}$ could have prevented us from detecting potential differences. Conversely, our data may likewise suggest the presence of potential reversible psychosocial distress in patients with TTS, since the patients participated in the study long after the acute event (median=44.6 \pm 20.2 months). Actually, research does not provide long-term follow-up on this topic.

\section{FC by RS-fMRI}

The estimation of the FC of the CAN during RS did not require precise a priori hypotheses derived from 
task-response effects in order to evaluate brain networks. This allowed a powerful exploratory screening of the functional organisation of the CAN.

Central neural abnormalities have been suggested to play a role in MVA and particularly in TTS. ${ }^{36-38}$ We did not observe any difference in the insular, amygdala or hippocampal CAN areas, which are likely implicated in the response to autonomic triggers in TTS. ${ }^{37}$ However, we found an increased functional integration between specific regions belonging to two physiologically distinct networks within the CAN characterised TTS long after the acute event. One could hypothesise that triggered functional differences in the limbic system may predispose patients to TTS acute manifestations, whereas a peculiar increased level of functional integration between sCAN-MCC and pCAN-PM areas in TTS under resting conditions may represent a predisposing substrate.

Actually, sCAN-MCC belongs to Brodmann area $24,{ }^{11}$ which is specifically involved in the interoceptive pain awareness, negative emotional statuses, pain avoidance and reward approach functions, all potentially implicated in chronic pain and obsessive-compulsive disorder; whereas, the pCAN-PM is a portion of the PM area involved in the parasympathetic regulation. ${ }^{39-41}$ Therefore, our data indicate that in patients with TTS there is a relatively stronger RS functional integration between brain regions involved in pain stimuli monitoring, emotional integration and visceral motor control, compared with patients with AMI. Recent retrospective studies have suggested that exposure not only to one acute stressful event, but also to chronic minor repetitive stressful conditions could contribute to development of TTS episodes. ${ }^{42}{ }^{43}$ It could be hypothesised that this long-term stress condition may increase vulnerability to TTS in susceptible subjects with RS functional CAN abnormalities.

Similarly, Sabisz et $a l^{44}$ showed a significant RS-fMRI signal difference in specific areas of the default mode network. Of note, recent structural MRI and fMRI data suggest that TTS might present a suboptimal anatomical organisation of the limbic system. ${ }^{38} 4546$ Differences in control group and in the time between the examination and the acute TTS episode (12-18 months vs $44.6 \pm 20.2$ in our study) may explain the disagreement with previously reported results. Therefore, we think this potential pathophysiological pathway deserves confirmation in a longitudinal study.

Moreover, we have investigated whether the peculiar functional organisation of the CAN in TTS correlates to specific psychological patterns, thus, linking psychosocial distress with central autonomic control of the cardiovascular system. However, we were not able to find a statistically significant correlation.

For the first time, we have reported RS-fMRI data in MVA. Rosen $e t a l^{36}$ showed activity of the right anteriorinsular cortex in patients with MVA using positron emission tomography during a positive dobutamine stress test. However, the patients with MVA in that study did not show any specific functional organisation of the CAN under resting conditions, thus, suggesting a triggered CAN dysfunction rather than functional predisposing abnormalities within the CAN. As for TTS, only a borderline negative correlation was found between the mFC of the sCAN-MCG and pCAN-PM and the perception of worse physical pain with the SF-36 BodyPain score.

\section{Limitations}

The sample size of the current study is the main limitation of the study, which predictably enrolled a majority of females. This limits the generalisation of the results and gender analysis. Moreover, MVA is manifested as chronic recurrent symptoms, whereas TTS and AMI are mostly acute events.

The cross-sectional design shares limitations with most of the studies on fMRI and psychiatric evaluation of patients with these conditions. Consequently, we cannot ascertain whether these specific findings are predisposing factors or consequences of the diseases. Nevertheless, the long interval between the acute event and the enrolment of patients with both TTS and AMI allowed us to exclude conceivable acute phase confounders.

Notably, the psychosocial and psychiatric evaluations may not be restricted to validated questionnaires and measurable variables. Nonetheless, these are measurable, easily administrable tools implemented in the current research and clinical practice.

Last, the study lacks of a matched-healthy control group. Notably, depression and anxiety are highly prevalent even in patients with CAD. ${ }^{47}$ Therefore, the small sample size and the higher prevalence of OCPT $(60 \%)$ than expected from clinical practice ${ }^{35}$ could prevent us to detect potential differences in psychosocial and psychiatric pattern by questionnaires. Similarly, limited sample size prevented us to perform any consistent sub-analysis comparing subjects with both TTS or MVA reporting emotional triggers and those not reporting them.

\section{CONCLUSIONS}

We implemented an innovative research collaboration among cardiologists, neuroscientists and psychiatrists ('Neuro-psycho-heart Team'). We hypothesise that complex mechanisms of somatisation may explain the discrepancy between self-reported mental health and the level of body pain in MVA. Moreover, TTS exhibits a stronger functional integration between two specific brain regions involved in pain experience regulation and visceromotor control. These data are hypothesis generating and provide novel suggestions for research strategies in this area, including pathophysiological investigations and researches on the potential effects of 
psychotherapy and stress-reducing techniques as therapeutic strategies.

\section{Author affiliations}

${ }^{1}$ Cardiovascular Research, Hospital of San Giovanni, Bellinzona, Switzerland

${ }^{2}$ Internal Medicine, Hospital of San Giovanni, Bellinzona, Switzerland

${ }^{3}$ Neuroradiology, Neurocenter of Southern Switzerland, Lugano, Switzerland

${ }^{4}$ Psychology, Psycho-Educational Center, Stabio, Switzerland

${ }^{5}$ Cardiology, Cardiocentro Ticino, Lugano, Switzerland

${ }^{6}$ Cardiology, University Heart Center, Zurich, Switzerland

${ }^{7}$ Cardiology, Catholic University of Sacred Heart, Rome, Italy

${ }^{8}$ Biostatistics, University of Applied Sciences and Arts of Southern Switzerland,

Lugano, Switzerland

${ }^{9}$ Psychiatry, Hospital Malcantonese Castelrotto, Castelrotto, Switzerland

${ }^{10}$ Cardiology, University of Zurich, Zurich, Switzerland

Contributors MMC, MC and AG contributed to the conception, planning, conduct, reporting, manuscript conception, drafting and final approval and are responsible for the overall content as guarantors. EP, MP and CC contributed to the planning, conduct, manuscript drafting and final approval. CL contributed to the statistical analysis, manuscript drafting and final approval. MM, AK, IS, LB, CG and FC contributed to the manuscript conception, drafting and final approval.Patients appreciated and supported an innovative collaboration between cardiologists, neuroscientists and psychiatrists working as a 'Neuro-psycho-heart Team', investigating their disorders. In this way, patients were involved in development of the research questions and aims. They were not involved in the other stages of the study.

Funding This study was funded by Swiss Heart Foundation.

Competing interests AG reports grants from the Swiss Heart Foundation and the ABREOC for unrelated work; IS reports grants from Swiss Heart Foundation and Swiss National Foundation for unrelated work.

Patient consent for publication Not required.

Ethics approval The study was conducted according to the principles of the Declaration of Helsinki, approved by the local ethics review board.

Provenance and peer review Not commissioned; externally peer reviewed.

Data availability statement Data may be obtained from a third party and are not publicly available.

Open access This is an open access article distributed in accordance with the Creative Commons Attribution Non Commercial (CC BY-NC 4.0) license, which permits others to distribute, remix, adapt, build upon this work non-commercially, and license their derivative works on different terms, provided the original work is properly cited, appropriate credit is given, any changes made indicated, and the use is non-commercial. See: http://creativecommons.org/licenses/by-nc/4.0/.

\section{ORCID iD}

Magdalena Maria Cattaneo http://orcid.org/0000-0001-5678-3460

\section{REFERENCES}

1 Lanza GA. Cardiac syndrome X: a critical overview and future perspectives. Heart 2007;93:159-66.

2 Prasad A, Lerman A, Rihal CS. Apical ballooning syndrome (TakoTsubo or stress cardiomyopathy): a mimic of acute myocardial infarction. Am Heart J 2008;155:408-17.

3 Altintas E, Yigit F, Taskintuna N. The impact of psychiatric disorders with cardiac syndrome $X$ on quality of life: 3 months prospective study. Int J Clin Exp Med 2014;7:3520-7.

4 Lutfi MF. Anxiety level and cardiac autonomic modulations in coronary artery disease and cardiac syndrome X patients. PLoS One 2017;12:e0170086.

5 Lamendola P, Lanza GA, Spinelli A, et al. Long-term prognosis of patients with cardiac syndrome X. Int J Cardiol 2010;140:197-9.

6 Delmas C, Lairez O, Mulin E, et al. Anxiodepressive disorders and chronic psychological stress are associated with Tako-Tsubo cardiomyopathy- new physiopathological hypothesis. Circ $J$ 2013;77:175-80

7 Summers MR, Lennon RJ, Prasad A. Pre-morbid psychiatric and cardiovascular diseases in apical ballooning syndrome (tako-tsubo/ stress-induced cardiomyopathy): potential pre-disposing factors? J Am Coll Cardiol 2010;55:700-1.
8 Pelliccia F, Parodi G, Greco C, et al. Comorbidities frequency in takotsubo syndrome: an international collaborative systematic review including 1109 patients. Am J Med 2015;128:654.e11-9.

9 Templin C, Ghadri JR, Diekmann J, et al. Clinical features and outcomes of takotsubo (stress) cardiomyopathy. N Engl J Med 2015;373:929-38.

10 Thayer JF, Lane RD. A model of neurovisceral integration in emotion regulation and dysregulation. J Affect Disord 2000;61:201-16.

11 Beissner F, Meissner K, Bär K-J, et al. The autonomic brain: an activation likelihood estimation meta-analysis for central processing of autonomic function. J Neurosci 2013;33:10503-11.

12 Spieker LE, Hürlimann D, Ruschitzka F, et al. Mental stress induces prolonged endothelial dysfunction via endothelin-A receptors. Circulation 2002;105:2817-20.

13 Lanza GA, Crea F. Primary coronary microvascular dysfunction: clinical presentation, pathophysiology, and management. Circulation 2010;121:2317-25.

14 Kurisu S, Kihara Y. Tako-tsubo cardiomyopathy: clinical presentation and underlying mechanism. J Cardiol 2012;60:429-37.

15 Pravatà E, Sestieri C, Mantini D, et al. Functional connectivity MR imaging of the language network in patients with drug-resistant epilepsy. AJNR Am J Neuroradiol 2011;32:532-40.

16 Pravatà $\mathrm{E}$, Zecca $\mathrm{C}$, Sestieri $\mathrm{C}$, et al. Hyperconnectivity of the dorsolateral prefrontal cortex following mental effort in multiple sclerosis patients with cognitive fatigue. Mult Scler 2016;22:1665-75.

17 Thygesen K, Alpert JS, Jaffe AS, et al. Third universal definition of myocardial infarction. Eur Heart J 2012;33:2551-67.

18 Ong P, Camici PG, Beltrame JF, et al. International standardization of diagnostic criteria for microvascular angina. Int $\mathrm{J}$ Cardiol 2018;250:16-20.

19 Ghadri J-R, Wittstein IS, Prasad A, et al. International expert consensus document on takotsubo syndrome (Part I): clinical characteristics, diagnostic criteria, and pathophysiology. Eur Heart $J$ 2018;39:2032-46.

20 Mazzi F, Stieglitz RD, Trabert W, et al. AMDP-8. Manuale per la metodologia e la documentazione della diagnosi in psichiatria / manual for the assessment and documentation of psychopathology /Arbeitsgemeinschaft für Methodik und Dokumentation in der Psychiatrie. Florence, Italy: Giunti Editore, 2011.

21 Boyle GJ, Le Déan L. Discriminant validity of the illness behavior questionnaire and Millon clinical Multiaxial Inventory-III in a heterogeneous sample of psychiatric outpatients. J Clin Psychol 2000;56:779-91.

22 Spielberger CD, Sydeman SJ. State-Trait anxiety inventory and State-Trait anger expression Inventory. The use of psychological testing for treatment planning and outcome assessment. Hillsdale, NJ: Lawrence Erlbaum Associates, 1994.

23 Ware JE, Sherbourne CD. The MOS 36-item short-form health survey (SF-36). I. conceptual framework and item selection. Med Care 1992;30:473-83.

24 Grös DF, Antony MM, Simms LJ, et al. Psychometric properties of the State-Trait inventory for cognitive and somatic anxiety (STICSA): comparison to the State-Trait anxiety inventory (STAI). Psychol Assess 2007;19:369-81.

25 Apolone G, Mosconi P. The Italian SF-36 health survey: translation, validation and norming. J Clin Epidemiol 1998;51:1025-36.

26 Ogawa S, Lee TM, Nayak AS, et al. Oxygenation-sensitive contrast in magnetic resonance image of rodent brain at high magnetic fields. Magn Reson Med 1990;14:68-78.

27 Whitfield-Gabrieli S, Nieto-Castanon A. Conn: a functional connectivity toolbox for correlated and anticorrelated brain networks. Brain Connect 2012;2:125-41

28 Carter AR, Astafiev SV, Lang CE, et al. Resting interhemispheric functional magnetic resonance imaging connectivity predicts performance after stroke. Ann Neurol 2010;67:365-75.

29 Lanza GA, Pasceri V, Colonna G, et al. Cardiac autonomic function and sensitivity to pain in postmenopausal women with angina and normal coronary arteries. Am J Cardiol 1997;79:1174-9.

30 Genovese CR, Lazar NA, Nichols T. Thresholding of statistical maps in functional neuroimaging using the false discovery rate. Neuroimage 2002;15:870-8.

31 Atienza F, Velasco JA, Brown S, et al. Assessment of quality of life in patients with chest pain and normal coronary arteriogram (syndrome X) using a specific questionnaire. Clin Cardiol 1999;22:283-91.

32 Daniel M, Agewall S, Caidahl K, et al. Effect of myocardial infarction with nonobstructive coronary arteries on physical capacity and quality-of-life. Am J Cardiol 2017;120:341-6.

33 Dickinson WP, Dickinson LM, deGruy FV, et al. The somatization in primary care study: a tale of three diagnoses. Gen Hosp Psychiatry 2003;25:1-7. 
34 Vermeltfoort IAC, Raijmakers PGHM, Odekerken DAM, et al. Association between anxiety disorder and the extent of ischemia observed in cardiac syndrome X. J Nucl Cardiol 2009;16:405-10.

35 Hueston WJ, Werth J, Mainous AG. Personality disorder traits: prevalence and effects on health status in primary care patients. Int $J$ Psychiatry Med 1999;29:63-74.

36 Rosen SD, Paulesu E, Wise RJS, et al. Central neural contribution to the perception of chest pain in cardiac syndrome X. Heart 2002;87:513-9.

37 Pereira VH, Marques P, Magalhães R, et al. Central autonomic nervous system response to autonomic challenges is altered in patients with a previous episode of takotsubo cardiomyopathy. Eur Heart J Acute Cardiovasc Care 2016;5:152-63.

38 Klein C, Hiestand T, Ghadri J-R, et al. Takotsubo Syndrome Predictable from brain imaging data. Sci Rep 2017;7:5434.

39 Vogt BA. Midcingulate cortex:structure, connections, homologies, functions and diseases. $J$ Chem Neuroanat 2016;74:28-46.

40 Tracey I, Mantyh PW. The cerebral signature for pain perception and its modulation. Neuron 2007;55:377-91.
41 Medford N, Critchley HD. Conjoint activity of anterior insular and anterior cingulate cortex: awareness and response. Brain Struct Funct 2010;214:535-49.

42 Rosman L, Dunsiger S, Salmoirago-Blotcher E. Cumulative impact of stressful life events on the development of takotsubo cardiomyopathy. Ann Behav Med 2017;51:925-30.

43 Wallström S, Ulin K, Määttä S, et al. Impact of long-term stress in takotsubo syndrome: experience of patients. Eur $\mathrm{J}$ Cardiovasc Nurs 2016;15:522-8.

44 Sabisz A, Treder N, Fijałkowska M, et al. Brain resting state functional magnetic resonance imaging in patients with takotsubo cardiomyopathy an Inseparable pair of brain and heart. Int $\mathrm{J}$ Cardiol 2016;224:376-81.

45 Hiestand T, Hänggi J, Klein C, et al. Takotsubo syndrome associated with structural brain alterations of the limbic system. J Am Coll Cardiol 2018;71:809-11.

46 Templin C, Hänggi J, Klein C, et al. Altered limbic and autonomic processing supports brain-heart axis in Takotsubo syndrome. Eur Heart J 2019;40:1183-7.

47 Huffman JC, Smith FA, Blais MA, et al. Recognition and treatment of depression and anxiety in patients with acute myocardial infarction. Am J Cardiol 2006;98:319-24. 